\title{
Digital droplet PCR for the detection and quantification of circulating bovine Deltapapillomavirus
}

\author{
Francesca De Falco ${ }^{1}$, Federica Corrado ${ }^{2}$, Anna Cutarelli ${ }^{2}$, LEONARDO LEONARDI $^{3}$, and \\ Sante Roperto ${ }^{4}$ \\ ${ }^{1}$ Universita degli Studi di Napoli Federico II Dipartimento di Medicina Veterinaria e \\ Produzioni Animali \\ ${ }^{2}$ Istituto Zooprofilattico Sperimentale del Mezzogiorno \\ ${ }^{3}$ University of Perugia- Faculty of Veterinary Medicine \\ ${ }^{4}$ Department of Veterinary Medicine
}

May 6, 2020

\begin{abstract}
In this study, the digital droplet polymerase chain reaction (ddPCR) was used to quantify circulating bovine papillomavirus (BPV; genus: Deltapapillomavirus) levels in blood samples from 25 healthy cows and 15 cows with chronic enzootic hematuria due to papillomavirus-associated bladder tumors. ddPCR detected the BPV DNA in $95 \%$ of all the samples (i.e., in 24 of the healthy cows and 14 of the diseased animals), whereas quantitative real time PCR (qPCR) detected it in only $57.5 \%$ of the same blood samples, with the percentage differences between ddPCR and qPCR being statistically significant (P value ? 0.05), according to the ?2 test of Campbell and Richardson. Furthermore, ddPCR detected BPV infections by a single genotype and by multiple genotypes in $37 \%$ and $63 \%$ of the cows, respectively, whereas qPCR detected these in $16 \%$ and $16 \%$, respectively. Of the two assays, ddPCR was the more sensitive and accurate clinical diagnostic tool, allowing the detection of otherwise undetectable BPV genotypes, and consequently, a higher number of BPV co-infections. qPCR failed to detect many BPV co-infections by multiple genotypes. Therefore, ddPCR may be an essential tool for improving diagnostic procedures, allowing the identification of the genotypic distribution of BPV and a better understanding about the territorial divergence, if any, of the BPV prevalence in different areas. No significant differences in the blood viral load estimations were observed between the two animal groups, suggesting that the bloodstream could be a site of primary infection. Finally, as BPV DNA was detected in cows affected by noninvasive urothelial tumors, including papilloma and papillary urothelial neoplasms of low malignant potential, the circulating BPVs appeared to be independent of the status of urothelial neoplasms. Therefore, unlike in humans, circulating BPVs cannot be an actual prognostic marker of urothelial tumors in cows.
\end{abstract}

Digital droplet PCR for the detection and quantification of circulating bovine Deltapapillomavirus Running title: ddPCR and BPV quantification in blood

Francesca De Falco ${ }^{1}$, Federica Corrado ${ }^{2}$, Anna Cutarelli $^{2}$, Leonardo Leonardi ${ }^{3}$, Sante Roperto ${ }^{1^{*}}$

${ }^{1}$ Dipartimento di Medicina Veterinaria e Produzioni Animali, Università degli Studi di Napoli

Federico II, Napoli, Italia.

${ }^{2}$ Istituto Zooprofilattico Sperimentale del Mezzogiorno, Portici, Napoli, Italia.

3 Dipartimento di Medicina Veterinaria, Università degli Studi di Perugia, Perugia, Italia.

*Corresponding author: e-mail address: 
sante.roperto@unina.it (SR)

Summary - In this study, the digital droplet polymerase chain reaction (ddPCR) was used to quantify circulating bovine papillomavirus (BPV; genus:Deltapapillomavirus ) levels in blood samples from 25 healthy cows and 15 cows with chronic enzootic hematuria due to papillomavirus-associated bladder tumors. ddPCR detected the BPV DNA in $95 \%$ of all the samples (i.e., in 24 of the healthy cows and 14 of the diseased animals), whereas quantitative real time PCR (qPCR) detected it in only $57.5 \%$ of the same blood samples, with the percentage differences between ddPCR and qPCR being statistically significant (P value [?] 0.05), according to the $\chi^{2}$ test of Campbell and Richardson. Furthermore, ddPCR detected BPV infections by a single genotype and by multiple genotypes in $37 \%$ and $63 \%$ of the cows, respectively, whereas qPCR detected these in $16 \%$ and $16 \%$, respectively. Of the two assays, ddPCR was the more sensitive and accurate clinical diagnostic tool, allowing the detection of otherwise undetectable BPV genotypes, and consequently, a higher number of BPV co-infections. qPCR failed to detect many BPV co-infections by multiple genotypes. Therefore, ddPCR may be an essential tool for improving diagnostic procedures, allowing the identification of the genotypic distribution of BPV and a better understanding about the territorial divergence, if any, of the BPV prevalence in different areas. No significant differences in the blood viral load estimations were observed between the two animal groups, suggesting that the bloodstream could be a site of primary infection. Finally, as BPV DNA was detected in cows affected by noninvasive urothelial tumors, including papilloma and papillary urothelial neoplasms of low malignant potential, the circulating BPVs appeared to be independent of the status of urothelial neoplasms. Therefore, unlike in humans, circulating BPVs cannot be an actual prognostic marker of urothelial tumors in cows.

Keywords: blood; bovine papillomavirus (BPV); droplet digital polymerase chain reaction (ddPCR); quantitative real time polymerase chain reaction (qPCR); chronic enzootic hematuria.

\section{Introduction}

Papillomaviruses (PVs) are small, non-enveloped, epitheliotropic, double-stranded DNA viruses that infect mucosal and cutaneous epithelia in a wide variety of animals in a species-specific manner (IARC, 2007). Bovine papillomaviruses (BPVs) consist of 28 genotypes that are distributed into five genera: Deltapapillomavirus (סPV) ( BPV-1, -2, -13, and -14), Xipapillomavirus ( $\chi \mathrm{PV})(\mathrm{BPV}-3,-4,-6,-9,-10,-11,-12,-15$, $-17,-20,-23,-24,-26$, and -28), Epsilonpapillomavirus (EPV) (BPV-5, -8, and -25),Dyokappapapillomavirus (DyoxPV) (BPV-16, -18, -22),Dyoxipapillomavirus (Dyo $\mathrm{PV}$ ) (BPV-7). BPV-19, -21, and -27 remain to be classified (http://pave.niaid.nih.gov/; Crespo et al., 2019).

Bovine $\delta \mathrm{PV}$ is generally considered to be associated with a high risk of neoplastic transformation (Daudt et al., 2018). Indeed, this BPV genus plays a central role in both cutaneous and bladder tumors of large ruminants (Campo et al., 1992; Roperto et al., 2013). In southern Italy, BPV-2 and -13 are the most important infectious agents involved in bladder carcinogenesis in Podolica breed cattle (Roperto et al., 2010; Roperto et al., 2016a).

Although bovine $\delta \mathrm{PVs}$ recognize Bos taurus as their classical host, this virus genus is the only one known to be characterized by natural cross-species transmission and infection (IARC, 2007). Bovine $\delta$ PVs cause cutaneous tumors in horses (Lancaster et al., 1979), buffaloes (Silvestre et al., 2009; Pangty et al., 2010; Somvanshi, 2011), cats (Munday et al., 2015), African lions (Orbell et al., 2011), and Cape mountain zebras, giraffes and sable antelopes (Williams et al., 2011). Bovine $\delta$ PV DNA has been identified in a squamous cell carcinoma involving the head and neck of a mare and in cutaneous wart lesions from sheep (Kainzbauer et al., 2012; Mazzucchelli-de-Souza et al., 2018).

The small-sized (40-85 amino acids) E5 protein, believed to be the major oncoprotein of $\delta \mathrm{PV}$, displays celltransforming activity via numerous pathways (DiMaio and Petti, 2013). The E6 protein of $\delta$ PVs appears to contribute to cell transformation through its interaction with the focal adhesion protein paxillin. With regard to the E7 protein, although it has no cell-transforming functionality by itself, the transformation process appears to be correlated with the protein's ability to bind to p600. 
Furthermore, PV infections are significantly associated with many adverse effects on the reproductive function, which may be related to the infecting genotype (Souho et al., 2015). A recent viral metagenomics analysis revealed a high prevalence of BPV-1 and -2 in the uterus of apparently heathy dairy cows (Ling et al., 2019). Furthermore, BPV DNA has been found in the uterus of healthy mares (Savini et al., 2019). $\mathrm{BPVs}$ are able to cause a congenital infection via transplacental transmission in cows and buffaloes (Roperto et al., 2019; Russo et al., 2020). Transplacental infections of BPV-2 and -13 in ewes have also been reported (Roperto et al., 2018).

There are few reports presenting quantification data of BPVs, and those that do are based on quantitative real-rime polymerase chain reaction (qPCR) analysis. Indeed, $\mathrm{qPCR}$ has for a long time been the method of choice for viral load estimation (Lillsunde Larsson and Helenius, 2017) and is considered the assay with the highest sensitivity and specificity for the detection of PV DNA and cDNA (Biron et al., 2016). To date, BPV-1 and -2 are the only genotypes that have been investigated by qPCR (Yuan et al., 2007; Cota et al., 2015).

The droplet digital polymerase chain reaction (ddPCR) is a robust PCR technique that is currently the most accurate and sensitive method for quantifying nucleic acids of interest. Unlike that by qPCR, quantifcation by ddPCR is absolute and does not require calibration of standards, thus rendering the process faster, more precise, and reproducible (Kanagal-Shamanna, 2017). Although ddPCR has not yet been reported for the detection of BPV, it is believed to have a potential higher accuracy than qPCR for detecting the virus. Sensitive and specific biomarkers for the blood-based analysis of analytes, such as viral DNA, as well as accurate molecular epidemiological tools, are still needed to achieve a superior diagnostic performance in identifying BPVs.

As there are currently no published studies on the quantification of circulating bovine $\delta \mathrm{PVs}$, the aim of the present study was to evaluate the performance of ddPCR in quantifying the viral load of high-risk genotypes $(1,2,13,14)$ of circulating bovine $\delta \mathrm{PV}$. The same blood samples were simultaneously assayed by qPCR in order to compare the diagnostic sensitivity of the two assays for this virus.

\section{Materials and Methods}

\subsection{Ethics statement}

The authors confirm that the ethical policies of the journal, as noted on the journal's Author Guidelines page, have been adhered to. No ethical approval was required for this study, as we did not perform any animal experiments. All blood samples were collected at public slaughterhouses. 2.2 Blood samples and DNA extraction Blood samples were harvested from 40 cows that had grazed on lands contaminated with bracken fern (Pteridium spp.) and collected into EDTA-containing anticoagulant tubes. Fifteen of these cows (3-18 years of age) had been suffering from chronic enzootic hematuria for several years. The other 25 cows (3-20 years of age) were healthy animals that had been in close contact with the diseased cows as they belonged to the same cattle breeding farm. These 25 cows were considered healthy as they did not exhibit any bladder lesions, which may be a clinical marker of BPV infection. The cows were slaughtered at public slaughterhouses located in three regions of southern Italy (Basilicata, Calabria, and Campania), according to the decision of the owners of the animals. Medical authorities had carried out an ante-mortem clinical examination of the animals according to national legislation. Total DNA was extracted using a DNeasy Blood \& Tissue Kit (Qiagen ${ }^{\mathrm{TM}}$, Wilmington, DE, USA), according to the manufacturer's instructions. 2.3 Digital droplet polymerase chain reaction

The simplex PCR designs were for four BPV genotypes (BPV-1, -2, -13, and -14). Table 1 reports the primers and probes used for the detection of these four genotypes as well as their GenBank accession numbers. A black hole quencher was used in combination with the fluorescent reporter dyes FAM and VIC. In brief, each PCR mixture (total volume $20 \mu \mathrm{L}$ ) contained $1 \mathrm{x}$ ddPCR ${ }^{\mathrm{TM}}$ Supermix for Probes (no dUTP; Bio-Rad, Mississaugua, ON, Canada) $0.9 \mu \mathrm{M}$ of the primer and $0.25 \mu \mathrm{M}$ of the probe (Applied Biosystems, Hilden, Germany) and $5 \mu \mathrm{L}$ of the DNA sample (100 ng). The Mastermix and DNA sample were thoroughly mixed and then transferred to a DG8 cartridge for placement in the QX100 Droplet Generator (Bio-Rad). Next, 
the Droplet Generation Oil for Probes (Bio-Rad) was added to the cartridge. After their generation, 40 $\mu \mathrm{L}$ of the droplets was carefully transferred to a twin.tec ${ }^{\mathrm{TM}}$ semi-skirted 96-well PCR plate (Eppendorf AG, Hamburg, Germany). The plate was sealed twice ( $4 \mathrm{~s}$ at $170{ }^{\circ} \mathrm{C}$ each time) using an Axygen Platemax semiautomated plate sealer (Thermo Fisher, Scientific, Waltham, MA, USA). Then, amplification was carried out in a T100 96-well thermal cycler (Bio-Rad). Two thermal gradients ranging from $57{ }^{\circ} \mathrm{C}$ to $62^{\circ} \mathrm{C}$ were tested to determine the optimal hybridization temperature of the assay. The PCR cycling conditions were as follows: enzyme activation at $95{ }^{\circ} \mathrm{C}$ for $10 \mathrm{~min} ; 40$ cycles of denaturation at $94{ }^{\circ} \mathrm{C}$ for $30 \mathrm{~s}$ and $56{ }^{\circ} \mathrm{C}$ for 1 min; enzyme deactivation at $98{ }^{\circ} \mathrm{C}$ for $10 \mathrm{~min}$; and a final hold at $12^{\circ} \mathrm{C}$. The droplets were quantified in a QX100 Droplet Reader (Bio-Rad) after which the ddPCR data were analyzed using Quantasoft Version 1.4. Manual thresholds were applied to both the BPV genotypes and the positive controls, the latter of which were BPV-1 DNA from a zebra sarcoid (Romanucci et al, 2020) (a kind gift provided by Dr. Borzacchiello, University of Naples, Italy); BPV-2 clone DNA (a kind gift by Dr A. Venuti, IRCSS Regina Elena, National Cancer Institute, Rome, Italy); and BPV-13 and -14 DNA from bovine bladder tumors from our laboratories (Roperto et al., 2016a, b). In each run, a BPV-negative sample and a non-template control were included. The BPV concentration was finally expressed as the number of copies of DNA per microliter of blood (copies $/ \mu \mathrm{L}$ ). Therefore, the PCR result could be directly converted into copies/ $\mu \mathrm{L}$ in the initial samples simply by multiplying it with the total volume of the reaction mixture $(22 \mu \mathrm{L})$ and then dividing that number by the volume of DNA sample added to the reaction mixture $(5 \mu \mathrm{L})$ at the beginning of the assay.

\subsection{Optimization of the digital droplet polymerase chain reaction assay of papillomaviruses}

The thermal gradient was tested in the range of $55-62{ }^{\circ} \mathrm{C}$ to determine the optimal hybridization temperature of the assay. The most suitable hybridization temperatures for the efficient detection of the bovine $\delta \mathrm{PVs}$ were $58{ }^{\circ} \mathrm{C}$ for BPV-1, -13 , and -14 and $56{ }^{\circ} \mathrm{C}$ for BPV-2. At these temperatures, the positive droplets showed the highest fluorescence amplitude and were well distinguishable from the negative droplets. To optimize the assay, three different DNA volumes were tested: 1,4 , and $5 \mu \mathrm{L}$. The results showed that increasing the DNA volume added to the PCR mixture increased the number of DNA copies detected, without complete inhibition of the reaction. As the aim of the ddPCR assay in this study was also to investigate the limit of detection of $\delta \mathrm{PVs}$ in low-level contaminated samples, the final DNA volume chosen was $5 \mu \mathrm{L}$, which corresponded to the highest volume of DNA that could be added to the ddPCR mixture in this study. The threshold was manually set at the fluorescence amplitude for each ddPCR run.

\subsection{Quantitative real-time polymerase chain reaction}

The qPCR assays were performed on the CFX96 Real-Time System of the C1000 Touch ${ }^{\mathrm{TM}}$ Thermal Cycler (Bio-Rad), using 96-well plates (Hard-Shell@ 96-Well PCR Plates, \#hsp9601; Bio-Rad). The following thermal cycling program was used: $50{ }^{\circ} \mathrm{C}$ for $2 \mathrm{~min}, 95{ }^{\circ} \mathrm{C}$ for $10 \mathrm{~min}$, and 40 cycles of $95{ }^{\circ} \mathrm{C}$ for $15 \mathrm{~s}$ and 58 ${ }^{\circ} \mathrm{C}$ for $60 \mathrm{~s}$. The reaction mixture (final volume $20 \mu \mathrm{L}$ ) contained 1x TaqMan Universal Master Mix (Applied Biosystems), $900 \mathrm{nM}$ of each of the forward and reverse primers, $250 \mathrm{nM}$ of the probe (Table 1), and $5 \mu \mathrm{L}$ of the DNA sample. Each sample was amplified in duplicate, and three independent PCR runs were carried out to obtain nine $\mathrm{Ct}$ values for the sample. The data acquisition and data analyses were performed using CFX Maestro $^{\mathrm{TM}}$ (Bio-Rad). The Ct values were determined using regression analysis, where a value of [?] 38 was considered to be a negative result, according to the cutoff indicated by Harper et al. (2010).

\subsection{Statistical analysis}

Differences in the proportions of detected cases were tested using the $\chi^{2}$ test of Campbell and Richardson, with P [?] 0.05 indicating statistical significance. All analyses were carried out using the R System. According to Damerla et al. (2019), the sensitivity of the assay was calculated as the number of blood samples positive for circulating BPV DNA divided by the total number of examined cows $(\mathrm{N}=40)$.

\section{Results}

ddPCR detected BPV DNA in 95\% (38/40) of the blood samples tested, whereas qPCR detected the virus in $57.5 \%(23 / 40)$ of the same samples, with the percentage difference being of statistical significance ( $\mathrm{P}$ 
[?] 0.05). Specifically, the BPV genotypes were identified in 24 of the $25(96 \%)$ healthy cows without any bladder lesions and in 14 of the 15 ( $93 \%)$ cows affected by naturally occurring PV-associated bladder tumors leading to chronic hematuria $(\mathrm{P}=0.68)$. Furthermore, ddPCR revealed a single genotype in $37 \%$ (14/38), and multiple genotypes in $63 \%(24 / 38)$, of the cows harboring BPV DNA. qPCR failed to detect the BPV DNA in samples tested negative by ddPCR. In fact, qPCR detected BPV DNA in only 6 ( $16 \%$ ) of the 14 samples detected by ddPCR to be infected with a single BPV genotype. Of the BPV co-infections detected by ddPCR, 13 ( $54 \%$ ) were by two genotypes, 8 ( $33 \%)$ were by three genotypes and $3(12.5 \%)$ were by four genotypes. By contrast, qPCR revealed BPV co-infections by multiple genotypes in only six samples; namely, four samples with co-infection caused by two genotypes, and two samples with co-infection caused by three and four genotypes, respectively. In particular, qPCR failed to detect co-infection by two genotypes in 10 of the 13 samples revealed by ddPCR. Furthermore, qPCR found only two BPV genotypes in a sample in which ddPCR detected four genotypes. Neither ddPCR nor qPCR were able to detect a single infection by BPV-1. Tables 2 summarizes these results, whereas Table 3 displays the BPV genotypic frequencies.

With regard to single and multiple BPV infections, BPV-13 was the most prevalent genotype as it was detected in $82 \%(31 / 38)$ of the samples by ddPCR and in $37 \%(14 / 38)$ of the samples by qPCR, with the percentage difference between the two assays being statistically significant $(\mathrm{P}=0.005)$. BPV-2 was the second most-common genotype, being detected in $53 \%(20 / 38)$ of the samples by ddPCR and in $24 \%$ $(9 / 38)$ of the samples by qPCR $(\mathrm{P}=0.001)$. BPV-14 was detected in $40 \%(16 / 38)$ of the samples by ddPCR and in $13 \%(5 / 38)$ of the samples by qPCR $(\mathrm{P}=0.005)$. Finally, BPV-1 was the least common of the four genotypes being found in a limited number of samples. Indeed, BPV-1 was detected in $24 \%(9 / 38)$ by ddPCR and $18 \%(7 / 38)$ by qPCR $(\mathrm{P}=0.52)$.

ddPCR was also used for quantification of BPVs, with the results showing clear distinctions between positive FAM (blue), and VIC (green) and negative (gray) droplets (Figures 1-4). There were also differences in the fluorescence amplitude range of the background (negative) droplets among the $\delta \mathrm{PV}$ samples; that is 30008000 for BPV-1, 4000-9000 for BPV-2, 2500-5000 for BPV-13, 600-2000 for BPV-14. The numbers of BPV DNA copies per microliter of blood in the samples from the 24 healthy cows and the 14 cows suffering from $\mathrm{PV}$-associated bladder tumors are summarized in Table 4.

\section{Discussion}

To the best of our knowledge, this is the first study that has performed both a qualitative and a quantitative estimation of circulating BPV DNA using the sensitive ddPCR approach. The BPV E5 DNA was detected in $95 \%$ of the blood samples tested (38/40); in particular, single or multiple BPV genotypes were detected and quantified in 14 of the 15 cows suffering from naturally occurring PV-associated, noninvasive, papillary urothelial tumors and in 24 of the 25 healthy cows belonging to the same cattle herd as the tumor-carrying animals. Both groups of cows shared the same grazing lands. The ddPCR assay quantified the number of E5 DNA copies per microliter of blood samples of all four bovine $\delta \mathrm{PV}$ genotypes. Our study showed that, as for human samples (Cheung et al., 2019), ddPCR could be a diagnostic procedure capable of detecting otherwise undetectable BPVs by liquid biopsy. To date, the prevalence and burden of bovine $\delta \mathrm{PV}$ have been investigated by PCR and qPCR only. A BPV-2 prevalence of $50 \%$ and $10 \%$ in blood from cattle with and without BPV-associated neoplastic bladder lesions, respectively, has been reported using semi-nested PCR (Wosiaki et al., 2005). In addition, Roperto et al. (2008) reported a 78\% and 39\% prevalence of BPV-2 in blood from cattle with urinary bladder tumors and from healthy cattle, respectively. High percentages of BPV-1 and -2 in the blood of cattle suffering from cutaneous papillomatosis have also been revealed by PCR (Diniz et al., 2009; Santos et al., 2016). Furthermore, in veterinary medicine, a few BPV detection and quantification studies have been performed using qPCR. For example, Yuan et al. (2007) reported quantitative data about BPV-1 and -2 in equine sarcoids and bovine bladder tumors, respectively. Pathania et al. (2012) studied the BPV-2 load in urinary bladder tumors from cows suffering from enzootic bovine hematuria (EBH), and Cota et al. (2015) reported quantitative data on BPV-2 infection in bovine urinary bladder lesions. In addition, Pangty et al. (2010) and Kumar et al. (2015) quantified the BPV-1, -2 and 
-5 DNA copy numbers in some cutaneous warts and wart-like lesions of the upper gastrointestinal tract in cattle and buffaloes.

We compared the diagnostic sensitivity of ddPCR with that of qPCR in evaluating the same liquid biopsy. In our study, ddPCR showed superior sensitivity compared with qPCR, the latter so far believed to be the gold standard for measuring PV DNA (Isaac et al., 2017). All percentage differences in the detection of circulating BPV E5 DNA between these two diagnostic procedures were of statistical significance. ddPCR appeared to be an assay that would allow for the remarkably improved diagnosis of BPV infections in comparison with both PCR and qPCR. Therefore, our results suggest that ddPCR is by far the most sensitive and accurate assay for BPV detection, in accordance with its recently shown performance for oncogenic human papillomavirus (HPV) detection (Biron et al., 2016).

No statistically significant differences in the viral load estimations were found between the blood samples from healthy and bladder tumor-affected cows, suggesting that circulating BPVs represent a primary blood infection rather than being the consequence of virus release from bladder tumors. Therefore, it is conceivable that BPVs can spread through the blood to infect all organs of the cow, including the bladder, thereby representing the major pathway responsible for a latent bladder infection by the virus. However, it is also possible that other minor pathways may occur, such as a secondary infection deriving from the paragenital area harboring the BPV infection (Campo, 2006). Although PVs are strictly species-specific, experimental data showing that these viruses can produce infection via the bloodstream in some animal models appear to strengthen our suggestions (Cladel et al., 2019). Furthermore, it is believed that blood could be an actual route of HPV dissemination as the virus has been found in peripheral blood mononuclear cells from healthy blood donors (Bodaghi et al., 2005; Chen et al., 2009; Vergara et al., 2019). A further biological feature characterizing BPV-associated bladder carcinoma would seem to corroborate our suggestion. It has been suggested that the circulating viral DNA detectable in human patients reflects the aggressive biological behavior of HPV-associated carcinoma thus representing a prognostic tumor biomarker (Jeannot et al., 2016; Damerla et al., 2019; Veyer et al., 2019). BPV-associated bladder carcinomas of cattle have a relatively low metastatic potential, as only 8-10\% of them migrate to distant organs (Pamukcu, 1974; Roperto et al., 2010). We detected circulating BPV DNA in cows affected by noninvasive urothelial tumors, including papilloma and papillary urothelial neoplasms of low malignant potential, which showed that the circulating BPVs appeared to be independent of the status of urothelial neoplasms. Therefore, circulating BPVs cannot be used as an actual prognostic biomarker of urothelial tumors in cattle.

ddPCR may be an essential tool for improving diagnostic procedures, helping us to accurately identify the genotypic distribution of BPV and to better understand the territorial divergence, if any, of the BPV prevalence in different areas and allowing us to gain insights into the molecular and ecological epidemiology of infectious diseases, including viral ones. In particular, ddPCR may be a better choice than qPCR for clinical application being able to detect otherwise undetectable PV infections and co-infections. This baseline information is necessary before any decision about livestock health management can be made. ddPCR can provide great advantages toward informing on the necessary measures to adopt in order to reduce the risk of BPV infection and/or co-infection and improving our knowledges about the molecular mechanisms of the disease. Of note, whether PV co-infections increase the risk of disease is not clear, as controversial results exist (Chen et al., 2019). Indeed, it has been suggested that PV co-infections are prone to increase the risk of disease, as multiple PV genotypes might act synergistically (Trottier et al., 2006). Recently, it was shown that superinfection exclusion may occur during PV co-infections, whereby a PV genotype would be able to block or exclude other PV genotypes, as was described for many other viruses (Biryukov and Meyers, 2018).

In conclusion, ddPCR provides new perspectives for the clinical diagnosis of BPV infections. Observational studies using diagnostic tools, such as ddPCR, are required to gain further insights into the biology of BPVs. Moreover, these studies could verify the role of BPVs also as non-oncogenic infectious agents. In this context, there is an urgent need to study whether there are relationships between BPV infections and novel molecular mechanisms that underlie emerging non-oncogenic diseases, including the reproductive disorders that are a major problem in farm animals. The accurate interpretation of such association will have significant 
implications for the therapeutic, prognostic, and management considerations in cattle breeding and general animal husbandry.

Acknowledgments: The authors wish to thank Dr G. Borzacchiello, Department of Veterinary Medicine and Animal Productions, Naples University, and Dr A. Venuti, IRCSS Regina Elena National Cancer Institute, Rome, for providing BPV-1 and BPV-2 positive samples as a kind gift; Dr G. Salvatore of the Regione Basilicata, Dr P. Sarnelli of the Regione Campania, and Dr S. Morace of the University of Catanzaro "Magna Graecia" for their technical help.

Funding: The authors disclosed receipt of the following financial support for the research, authorship, and/or publication of this article: This project was partly supported by grants from the Regione Basilicata and the Regione Campania.

Declaration of Conflicting Interests; The authors declared no potential conflicts of interest with respect to the research, authorship, and/or publication of this article.

Data Availability Statement: All data supporting this manuscript are reported and can be found in our paper.

\section{References}

Biron, V.L., Kostiuk, M., Isaac, A., Puttagunta, L., O’Connell, D.A., Harris, J., Côté, D.W., \& Seikaly, H. (2016). Detection of human papillomavirus type 16 in oropharyngeal squamous cell carcinoma using droplet digital polymerase chain reaction. Cancer, 122, 1544-1551. doi: 10.1002/cncr.29976.

Biryukov, J., \& Meyers, C. (2018). Superinfection exclusion between two high-risk human papillomavirus types during coinfection. Journal of Virology, 92, e01993-17. doi: 10.1128/JVI.01993-17.

Bodaghi, S., Wood, L.V., Roby, G., Ryder, C., Steinberg, S.M., \& Zheng, Z.M. (2005). Could human papillomaviruses be spread through blood? Journal of Clinical Microbiology, 43, 5428-5434. doi: 10.1128/JCM.43.11.5428.5434.2005.

Campo, M.S. (2006). Papillomavirus Research - from natural history to vaccine and beyond. Campo, M.S., Ed,; Caister Academic Press, Norfolk, England, pp 373-387.

Campo, M.S., Jarrett, W.F.H., Barron, R.J., O’Neil, B.W., \& Smith, K.T. (1992). Association of bovine papillomavirus type 2 and bracken fern with bladder cancer in cattle. Cancer Research, 52, 6898-6904.

Chen, A.C., Keleher, A., Kedda, M.A., Spurdle, A.B., McMillan, N.A., \& Antonsson, A. (2009). Human papillomavirus DNA detected in peripheral blood samples from healthy Australian male blood donors. Journal of Medical Virology, 81, 1792-1796. doi: 10.1002/jmv.21592.

Chen, Z., Li, Q., Huang, Q., Liu, H., Jiang, H., Chen, Z., An, Z., \& Luo, Q. (2019). Characteristics of human papillomaviruses distribution in Guizhou Province, China. Virology Journal, 16, 123. doi: 10.1186/s12985019-1239-0.

Cheung, T.H., Yim, S.F., Yu, M.Y., Worley Jr, M.J., Fiascone, S.J., Chiu, R.W.K., Lo, K.W.K., Siu, N.S.S., Wong, M.C.S., Yeung, A.C.M., Wong, R.R.Y., Chen, Z.G., Elias, K.M., Chung, T.K.H., Berkowitz, R.S., Wong, Y.F., \& Chan, P.K.S. (2019). Liquid biopsy of HPV DNA in cervical cancer. Journal of Clinical Virology, 114, 32-36. doi: 10.1016/j.jcv.2019.03.005.

Cladel, N.M., Jiang, P., Li, J.J., Peng, X., Cooper, T.K., Majerciak, V., Balogh, K.K., Meyer, T.J., Brendle, S.A., Budgeon, L.R., Shearer, D.A., Munden, R., Cam, M., Vallur, R., \& Christensen, N.D., Zheng, Z.M., Hu, J. (2019). Papillomavirus can be transmitted through the blood and produce infections in blood recipients: Evidence from two animal models. Emerging Microbes \& Infections, 8, 1108-1121. doi: 10.1080/22221751.2019.1637072.

Cota, J.B., Peleteiro, M.C., Petti, L., Tavares, L., \& Duarte, A. (2015). Detection and quantification of bovine papillomavirus type 2 in urinary bladders and lymph nodes in cases of Bovine Enzootic Hematuria 
from the endemic region of Azores. Veterinary Microbiology, 178, 138-143. doi: 10.1016/j.vetmic.2015.03.026.

Crespo, SEI., Lunardi, M., Otonel, R.A.A., Headley, S.A., Alfieri, A.F., \& Alfieri, A.A. (2019). Genetic characterization of a putative new type of bovine papillomavirus in the Xipapillomavirus 1 species in a Brazilian dairy herd. Virus Genes , 55, 682-687. doi: 10.1007/s11262-019-01694-8.

Damerla, R.R., Lee, N.Y., You, D., Soni, R., Shah, R., Reyngold, M., Katabi, N., Wu, V., McBride, S.M., Tsai, C.J., Riaz, N., Powell, S.N., Babady, N.E., Viale, A., \& Higginson, D.S. (2019). Detection of early human papillomavirus-associated cancers by liquid biopsy. JCO Precision Oncology, 3, doi:10.1200/PO.18.00276.

Daudt, C., da Silva, F.R., Streck, A.F., Weber, M.N., Mayer, F.Q., Cibulski, S.P., \& Canal, C.W., (2016). How many papillomavirus species can go undetected in papilloma lesions? Scientific Reports, 6 :36480. doi $10.1038 /$ srep36480.

DiMaio, D., \& Petti, E. (2013). The E5 proteins. Virology, 445, 99-144. doi:10.1016/j.virol.2013.05.006.

Diniz, N., Melo, T.C., Mori, E., Brandão, P.E., Richtzenhain, Freitas, A.C., Beçak, W., Carvalho, R.F., \& Stocco, R.C. (2009). Simultaneous presence of bovine papillomavirus in blood and in short-term lymphocyte cultures from dairy cattle in Pernambuco, Brazil. Genetics and Molecular Research, 8, 1474-1480. doi: $10.4238 /$ vol8-4gmr668.

Harper, S.J., Ward, L.I., \& Clover, G.R. (2010). Development of LAMP and real-time PCR methods for the rapid detection of Xilellafastidiosa for quarantine and field applications. Phytopathology,100, 1282-1288. doi: 10.1094/PHYTO-06-10-0168.

Isaac, A., Kostiuk, M., Zhang, H., Lindsay, C., Makki, F., O'Connell, D.A., Harris, J.R., Cote, W.D.J., Seikaly, H., \& Biron, V.L. (2017). Ultrasensitive detection of oncogenic human papillomavirus in oropharyngeal tissue swabs. Journal of Otolaryngology - Head and Neck Surgery, 46, 5. doi: 10.1186/s40463-016-0177-8.

Jeannot, E., Becette, V., Campitelli M., Calméjane, M.A., Lappartient, E., Ruff, E., Saada, S., Holmes, A., Bellet, D., \& Sastre-Garau, X. (2016). Circulating human papillomavirus DNA detected using droplet digital PCR in the serum of patients diagnosed with early stage human papillomavirus-associated invasive carcinoma. The Journal of Pathology: Clinical Research, 2, 201-209. doi: 10.1002/cjp2.47.

Kainzbauer, C., Rushton, J., Tober, R., Scase, T., Nell, B., Sykora, S., \& Brandt, S. (2012). Bovine papillomavirus type 1 and Equus caballus papillomavirus 2 in equine squamous cell carcinoma of the head and neck in a Connemara mare. Equine Veterinary Journal , 44, 112-115. doi:10.1111/j.2042-3306.2010.00358.x.

Kanagal-Shamanna, R. (2016) Digital PCR: Principles and Applications. Methods in Molecular Biology, 1392, 43-50. doi: 10.1007/978-1-4939-3360-0_5.

Kumar, P., Nagarajan, N., Saikumar, G., Arya, R.S., \& Somvanshi, R. (2015). Detection of bovine papilloma viruses in wart-like lesions of upper gastrointestinal tract of cattle and buffaloes. Transboundary and Emerging Diseases, 62, 264-271. doi: 10.1111/tbed.12127.

Lancaster, W.D., Theilen, G.H., \& Olson, C. (1979). Hybridization of bovine papilloma virus type 1 and type 2 DNA to DNA from virus-induced hamster tumors and naturally occurring equine tumors. Intervirology, 11, 227-233. doi: 10.1159/000149038.

Lillsunde Larsson, G., \& Helenius, G. (2017). Digital droplet PCR (ddPCR) for the detection and quantification of HPV 16, 18, 33 and 45 - a short report. Cell Oncology, 40, 521-527. doi: 10.1007/s13402-017-0331-y.

Ling, Y., Zhang, X., Qi, G., Yang, S., Jingjlao, L., Shen, Q., Wang, X., Cui, L., Hua, X., Deng, X., Delwart, E., Zhang, W. (2019). Viral metagenomics reveals significant viruses in the genital tract of apparently healthy dairy cows. Archives of Virology 164, 1059-1067. doi:10.1007/s00705-019-04158-4.

Mazzuchelli-de-Souza, J., de Carvalho, R.E., Módolo, D.G., Thompson, C.E., Araldi, R.P., \& Stocco, R.C. First detection of bovine papillomavirus type 2 in cutaneous wart lesions from ovines. Transboundary and Emerging Diseases, 65, 939-943. doi: 10.1111/tbed.12892. 
Munday, J.S., Thomson, N., Dunowska, M., Knight, C.G., Laurie, R.E., \& Hills, S. (2015). Genomic characterisation of the feline sarcoid-associated papillomavirus and proposed classification as Bos taurus papillomavirus type 14. Veterinary Microbiology, 177, 289-295. doi: 10.1016/j.vetmic.2015.03.019.

Orbell, G.M., Young, S., \& Munday, J.S. (2011). Cutaneous sarcoids in captive African lions associated with feline sarcoid-associated papillomavirus infection. Veterinary Pathology, 48, 1176-1179. doi: 10.1177/0300985810391111.

Pamukcu, A.M. (1974). Tumours of the urinary bladder. Bulletin of the World Health Organization 50, 43-52.

Pangty, K., Singh, S., Goswami, R., Saikumar, G., \& Somvanshi, R. (2010). Detection of BPV-1 and -2 and quantification of BPV-1 by real-time PCR in cutaneous warts in cattle and buffaloes. Transboundary and Emerging Diseases, 57 , 185-196. doi: 10.1111/j.1865-1682.2009.01096.x.

Pathania, S., Dhama, K., Saikumar, G., Shahi, S., \& Somvanshi, R. (2012). Detection and quantification of bovine papilloma virus type 2 (BPV-2) by real-time PCR in urine and urinary bladder lesions in enzootic bovine haematuria (EBH)-affected cows. Transboundary and Emerging Diseases, 59, 79-84. doi: 10.1111/j.1865-1682.2011.01248.x.

Papillomavirus Episteme (PaVE), 2017 -http://pave.niaid.nih.gov/.

Romanucci, M., Borzacchiello, G., Defourny, S.V.P., Corvini, A., Altamura, G., Petrizzi, L., Della Salda, L. (2020). Fibroblastic sarcoid in a Grant's Zebra in Europe and its association with bovine papillomavirus type 1. Journal of Exotic Pet Medicine, 32, 54-55. doi: 10.1053/j.jepm.2019.12.002.

Roperto, S., Borzacchiello, G., Brun, R., Leonardi, L., Maiolino, P., Martano, M., Paciello, O., Papparella, S., Restucci, B., Russo, V., Salvatore, G., Urraro, C., \& Roperto, F. (2010) A review of bovine urothelial tumours and tumour-like lesions of the urinary bladder. Journal of Comparative Pathology, 142, 95-108. doi: 10.1016/j.jcpa.2009.08.156.

Roperto, S., Brun, R., Paolini, F., Urraro, C., Russo, V., Borzacchiello, G., Pagnini, U., Raso, C., Rizzo, C., Roperto, F., \& Venuti, A. (2008). Detection of bovine papillomavirus type 2 in the peripheral blood of cattle with urinary bladder tumours: possible biological role. Journal of General Virology 89, 3027-3033. doi: 10.1099/vir.0.2008/004457-0.

Roperto S, Munday JS, Corrado F, Goria M, Roperto F. (2016b). Detection of bovine papillomavirus type 14 DNA sequences in urinary bladder tumors of cattle. Veterinary Microbiology 190, 1 -4. doi: 10.1016/j.vetmic.2016.04.007.

Roperto, S., Russo, V., Corrado, F., De Falco, F., Munday, J.S., \& Roperto, F. (2018). Oral fibropapillomatosis and epidermal hyperplasia of the lip in newborn lambs associated with bovine Deltapapillomavirus. Scientific Reports, 8, 13310. doi: 10.1038/s41598-018-31529-9.

Roperto, S., Russo, V., De Falco, F., Taulescu, M., \& Roperto, F. (2019). Congenital papillomavirus infection in cattle: evidence for transplacental transmission. Veterinary Microbiology, 230, 95-100. doi:10.1016/j.vetmic.2019.01.019.

Roperto S, Russo V, Leonardi L, Martano, M., Corrado, F., Riccardi, M.G., \& Roperto, F. (2016a). Bovine papillomavirus type 13 expression in the urothelial bladder tumours of cattle. Transboundary and Emerging Diseases, 63, 628-634. doi: 10.1111/tbed.12322.

Roperto, S., Russo, V., Ozkul, A., Sepici-Dincel, A., Maiolino, P., Borzacchiello, G., Marcus, I., Esposito, I., \& Riccardi, M.G. (2013). Bovine papillomavirus type 2 infects the urinary bladder of water buffalo (Bubalus bubalis) and plays a crucial role in bubaline urothelial carcinogenesis. Journal of General Virology, 94, 403-408. doi: 10.1099/vir.0.047662-0.

Russo, V., Roperto, F., De Biase, D., Cerino, P., Urraro, C., Munday, J.S., \& Roperto, S. (2020). Bovine papillomavirus type 2 infection associated with papillomatosis of the amniotic membrane in water buffaloes 
(Bubalus bubalis). Pathogens, 9, 262. doi:10.3390/pathogens9040262.

Santos, E.U.D., Silva, M.A.R., Pontes, N.E., Coutinho, L.C.A., Paiva, S.S.L., Castro, R.S., \& Freitas, A.C. (2016). Detection of different bovine papillomavirus types and co-infection in bloodstream of cattle.Transboundary and Emerging Diseases, 63, e103-e108. doi: 10.1111/tbed.12237.

Savini, F., Gallina, L., Mazza, F., Mariella, J., Castagnetti, C., \& Scagliarini, A. (2019). Molecular detection of bovine papillomavirus DNA in the placenta and blood of healthy mares and respective foals. Veterinary Sciences, 6, 14. doi: 10.3390/vetsci6010014.

Silvestre, O., Borzacchiello, G., Nava, D., Iovane, G., Russo, V., Vecchio, D., D'Ausilio, F., Gault, F., Campo, M.S., \& Paciello, O. (2009). Bovine papillomavirus type 1 DNA and E5 oncoprotein expression in water buffalo fibropapillomas. Veterinary Pathol ogy, 46, 636-641. doi: 10.354/vp.09-VP-0222-P-FL.

Somvanshi, R. (2011). Papillomatosis in buffaloes: a less-known disease. Transboundary and Emerging Diseases, 58, 327-332. doi: 10.1111/j.1865.1682.2011.01211.x.

Souho, T., Benlemilih, M., \& Bennani, B. (2015). Human papillomavirus infection and fertility alteration: a systemic review. PLoS One,10:e0126936. doi: 10.1371/journal.pone.0126936.

Trottier, H., Mahmud, S., Costa, M.C., Sobrinho, J.P., Duarte-Franco, E., Rohan, T.E., Ferenczy, A., Villa, L.L., \& Franco, E.L., (2006). Human papillomavirus infections with multiple types and risk of cervical neoplasia. Cancer, Epidemiology, Biomarkers 83 Prevention, 15, 1274-1280. doi: 10.1158/1055-9965.EPI-060129 .

Vergara, N., Balanda, M., Vidal, D., Roldán, F., Martin, H.S., \& Ramírez, E. (2019). Detection and quantification of human papillomavirus DNA in peripheral blood mononuclear cells from donors. Journal of Medical Virology, 91, 2009-2015. doi: 10.1002/jmv.25551.

Veyer, D., Wack, M., Mandavit, M., Garrigou, S., Hans, S., Bonfils, P., Tartour, E., Bélec, L., Wang-Renault, S.F., Laurent-Puig, P., Mirghani, H., Rance, B., Taly, V., Badoual, C., \& Péré, H. (2019). HPV circulating tumoral DNA quantification by droplet-based digital PCR: a promising predictive and prognostic biomarker for HPV-associated oropharyngeal cancers. International Journal of Cancer,doi: 10.1002/ijc.32804.

Yuan, Z., Gallagher, A., Gault, E.A., Campo, M.S., \& Nasir, L. (2007). Bovine papillomavirus infection in equine sarcoids and in bovine bladder cancers. The Veterinary Journal, 174, 599-604. doi: 10.1016/j.tvjl.2006.10.012.

Williams, J.H., van Dyk, E., Nel, P.J., Lane, E., Van Wilpe, E., Bengis, R.G., de Klerk-Lorist, L.M., \& van Heerden, J. (2011). Pathology and immunohistochemistry of papillomavirus-associated cutaneous lesions in Cape mountain zebra, giraffe, sable antelope and African buffalo in South Africa. Journal of the South African Veterinary Association, 82, 97-106.

World Health Organization - IARC Monographs on the Evaluation of Carcinogenic Risks to Humans. (2007). Human papillomaviruses, vol. 90. Lyon, France, WHO Press.

Wosiaki, S.R., Barreiro, M.A., Alfieri, A.F., \& Alfieri, A.A. (2005). Semi-nested PCR for detection and typing bovine papillomavirus type 2 in urinary bladder and whole blood from cattle with enzootic haematuria. Journal of Virological Methods, 126, 215-219. doi: 10.1016/j.jviromet.2005.01.021.

Legends

Figure 1: Digital droplet polymerase chain reaction (ddPCR) for BPV-1 detection, shown for samples that were positive and negative for the E5 DNA of BPV-1. Blue droplets = positive droplets, gray droplets = negative droplets. QuantaSoft screenshots show the ddPCR results: A07 is a weakly positive sample; B07 through F07 are negative samples, and G07 is the positive control.

Figure 2: Digital droplet polymerase chain reaction (ddPCR) for BPV-2 detection, shown for samples that were positive and negative for the E5 DNA of BPV-2. Green droplets = positive droplets, gray droplets 
= negative droplets. QuantaSoft screenshots show the ddPCR results: A01, B01, C01 and F01 are positive samples, and the last sample is negative.

Figure 3: Digital droplet polymerase chain reaction (ddPCR) for BPV-13 detection, shown for samples that were positive and negative for the E5 DNA of BPV-13. Blue droplets = positive droplets, gray droplets = negative droplets. QuantaSoft screenshots show the ddPCR results: G01 is a negative sample, and the other samples are all positive.

Figure 4: Digital droplet polymerase chain reaction (ddPCR) for BPV-14 detection, shown for samples that were positive and negative for the E5 DNA of BPV-14. Blue droplets = positive droplets, gray droplets = negative droplets. QuantaSoft screenshots show the ddPCR results: A02 and B02 through E02 are weakly positive samples, and $\mathrm{C} 02$ and D02 are negative samples.
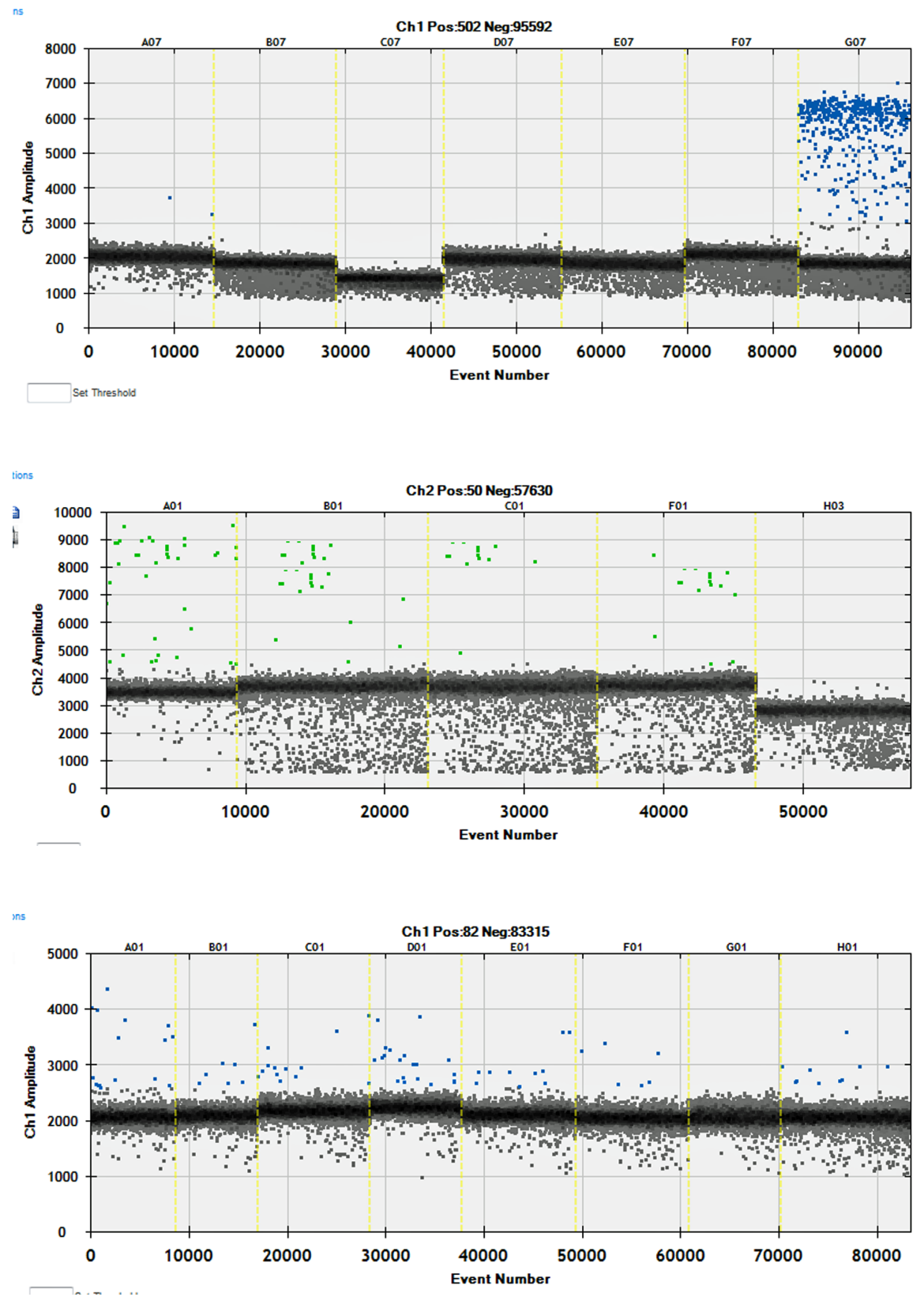


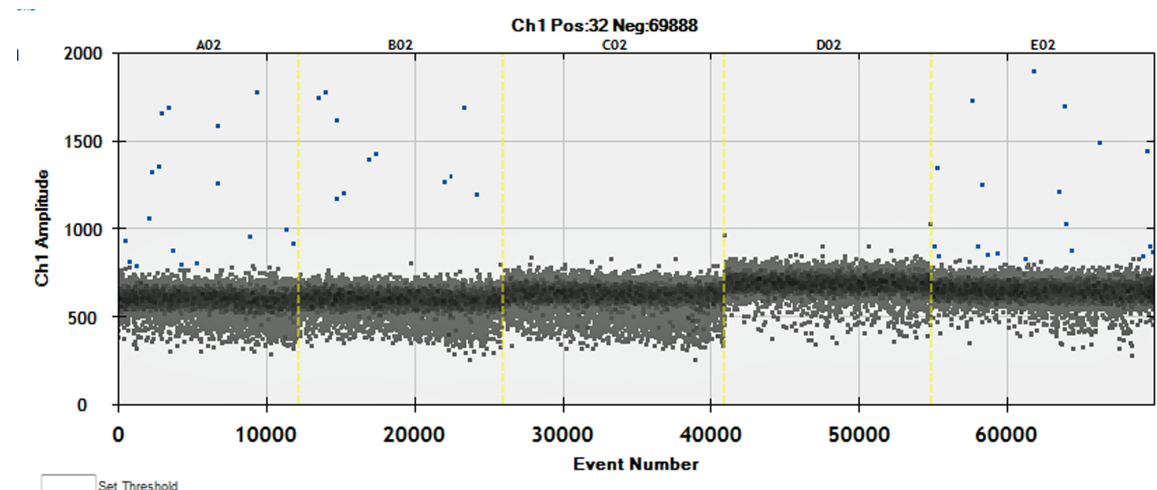

\section{Hosted file}

Table 1.docx available at https://authorea.com/users/319003/articles/448808-digital-dropletpcr-for-the-detection-and-quantification-of-circulating-bovine-deltapapillomavirus

\section{Hosted file}

Table 2.docx available at https://authorea.com/users/319003/articles/448808-digital-dropletpcr-for-the-detection-and-quantification-of-circulating-bovine-deltapapillomavirus

\section{Hosted file}

Table 3.docx available at https://authorea.com/users/319003/articles/448808-digital-dropletpcr-for-the-detection-and-quantification-of-circulating-bovine-deltapapillomavirus

\section{Hosted file}

Table 4.docx available at https://authorea.com/users/319003/articles/448808-digital-dropletpcr-for-the-detection-and-quantification-of-circulating-bovine-deltapapillomavirus 